This is an open access article distributed under the terms of the Creative Commons BY-NC-ND Licence

\title{
Genome-wide identification of circular RNAs in tomato seeds in response to high temperature
}

\author{
R. ZHOU ${ }^{1,2}$, X.Q. YU ${ }^{3}$, L.P. XU ${ }^{4}$, Y.L. WANG ${ }^{1,2}$, L.P. ZHAO ${ }^{1,2}$, T.M. ZHAO ${ }^{1,2 *}$, and W.G. YU ${ }^{1,2 *}$ \\ Institute of Vegetable Crop, Jiangsu Province Academy of Agricultural Sciences, \\ Jiangsu, 210014 Nanjing, P.R. China ${ }^{1}$ \\ Jiangsu Key Laboratory for Horticultural Crop Genetic Improvement, Jiangsu, 210014 Nanjing, P.R. China 2 \\ College of Horticulture, Nanjing Agricultural University, Jiangsu, 210095 Nanjing, P.R .China ${ }^{3}$ \\ Institute of Vegetable Research in Xining, Qinghai, 810000 Xining, P.R. China ${ }^{4}$
}

\begin{abstract}
Circular RNAs (circRNAs), an emerging class of non-coding RNAs, are abundant in eukaryotic transcriptomes. Seed germination is one of the most important stages in the entire life cycle of plants that can be slowed down or totally restrained by high temperature. Our aim is to identify heat-responsive circRNAs and explore the potential function of circRNAs in tomato seeds at high temperature. Following high-throughput sequencing, 4164 circRNAs were identified, and 980 circRNAs were shared in the control and high-temperature libraries. Among the 748 circRNAs with high expressions, 73 circRNAs were significantly up-/down- regulated in tomato seeds germinated at high temperature compared to the control. The parental genes of circRNAs existing in seeds only at high temperature were mainly involved in metabolic processes, cellular processes, catalytic activities, and binding based on Gene Ontology analysis. The results suggested that circRNAs were widespread in tomato and were generated from different chromosomes and diverse genomic regions. Some circRNAs in tomato seeds responded to high temperature during germination. This study provides the first genome-wide profile of circRNAs in response to high temperature during tomato seed germination and lays a foundation for studying the potential biological functions of circRNAs responding to heat stress.
\end{abstract}

Additional key words: chromosome distribution, heat stress, non-coding RNAs, seed germination, Solanum lycopersicum.

\section{Introduction}

In addition to messenger RNAs (mRNAs), there are diverse classes of non-coding RNAs in cells, including microRNAs (miRNAs), long non-coding RNAs (lncRNAs), and circular RNAs (circRNAs) (Ye et al. 2015, Zuo et al. 2016). CircRNAs primarily generate exons or introns in the nucleus, transfer to the cytoplasm by the nuclear pore complex, and function in the cytoplasm (Hansen et al. 2013, Memczak et al. 2013, Jeck et al. 2014). Previous studies found that circRNAs were not only abundant with stable expression but also played crucial roles in regulating a series of growth and development processes in animals (Jeck et al. 2012, Memczak et al.

2013, Guo et al. 2014, Meng et al. 2016). CircRNAs could promote the expression of their parental genes (Li et al. 2015). Also, CircRNAs and their linear forms might function as post-transcriptional regulators of their parental genes (Lu et al. 2015) in plants. Thus, CircRNAs with potential biological functions has become the latest research hotspot (Huang et al. 2015, Meng et al. 2016).

With the development of high-throughput sequencing, functional genomics and novel bioinformatic approaches, circRNAs have been widely identified in animals (Memczak et al. 2013, Guo et al. 2014, Jeck et al. 2014, Meng et al. 2016). However, there are few reports

Submitted 7 October 2017, last revision 25 May 2018, accepted 30 May 2018.

Abbreviations: circRNAs - circular RNAs; GO - Gene Ontology; miRNAs - microRNAs.

Acknowledgements: We acknowledge funding from the National Natural Science Foundation of China (31601745) and the Natural Science Foundation of Jiangsu Province (BK20160579). The authors thank Duanyue Huang and Chunfang Han for their assistance during the experiments.

* Corresponding authors; e-mails: tmzhaomail@163.com; wenguiyu@jaas.ac.cn. 
concerning circRNAs in plants, and the roles of circRNAs remain unclear in plants (Meng et al. 2016). To date, circRNA research has been carried out for Oryza sativa, Arabidopsis thaliana, and Solanum lycopersicum (Wang et al. 2014, Lu et al. 2015, Ye et al. 2015, Zuo et al. 2016). Ye et al. (2015) identified 12307 and 6012 circRNAs in Oryza sativa and Arabidopsis thaliana. Lu et al. (2015) found 2354 circRNAs in Oryza sativa. Zuo et al. (2016) identified 854 circRNAs in Solanum lycopersicum. CircRNAs are prevalent as crucial non-coding regulators in plants (Wang et al. 2014, Lu et al. 2015, Ye et al. 2015, Zuo et al. 2016).

Seed germination is the initial stage in the plant life cycle (Grime et al. 1991) and it may face different types of biotic and abiotic stresses (Jain et al. 2006, Guan et al. 2009, Fatemeh et al. 2010). Temperature, a crucial climatic factor, plays a principal role in influencing seed germination (Fyfield et al. 1989, Jain et al. 2006). Different species have varied temperature ranges, within which seed germination can occur and proceed (Jain et al. 2006, Fatemeh et al. 2010). High temperature may slow down or completely suppress seed germination, depending on the plant species and heat stress intensity (Wahid et al. 2007).

Tomato (Solanum lycopersicum L.) is not only a

\section{Materials and methods}

Plants and treatments: The seeds of Solanum lycopersicum L. cv. Sufen No. 14 were collected from the Jiangsu Province Academy of Agricultural Sciences in July 2016. The seeds were stored in paper bags at room temperature $\left(20 \pm 4{ }^{\circ} \mathrm{C}\right)$ and low air humidity (using lime powder as a drying agent) before germination.

The seeds were germinated in distilled water in Petri dishes (diameter $9 \mathrm{~cm}$ ) under darkness in climate chambers (RDN-560E-4, Dongnan Instrument Company, Ningbo, China). There were four temperature treatments, including $26{ }^{\circ} \mathrm{C}$ as a control and 32,36 , and $40{ }^{\circ} \mathrm{C}$ as high temperatures together with a $60 \%$ relative humidity, and a $\mathrm{CO}_{2}$ concentration of $400 \mu \mathrm{mol} \mathrm{mol}{ }^{-1}$. Forty seeds in one Petri dish were regarded as one replication and eight replications were used per treatment. Germination rates were calculated after 12, 24, 36, 48, and $60 \mathrm{~h}$. The seed was scored as germinated when a seed coat was broken and a radicle was visible. The analysis of variance (ANOVA) of the seed germination rate at different temperatures was carried out using SPSS 16.0 for Windows (SPSS, Chicago, IL, USA).

Circular RNA identification: Based on the results from the high-temperature test, the seeds were treated at $26^{\circ} \mathrm{C}$ as a control and $36^{\circ} \mathrm{C}$ as a high temperature in the climate chambers. After $24 \mathrm{~h}$ of incubation, the seeds from two Petri dishes at the same temperature were wiped dry and collected together to ensure that the sample quantity was globally important vegetable but also a model plant for scientific study (Mueller et al. 2005). The germination rate of tomato seeds was low at $35{ }^{\circ} \mathrm{C}$ and $40{ }^{\circ} \mathrm{C}$, and the seedlings that emerged from the seeds at $40{ }^{\circ} \mathrm{C}$ failed to develop further (Jain et al. 2006). Similarly, the germination of tomato seeds at $35{ }^{\circ} \mathrm{C}$ was significantly inhibited compared to the control (Wahid et al. 2007, Fatemeh et al. 2010).

However, there have been no reports concerning circRNA identification in tomato seeds at high temperature and their potential roles in the regulation of parental genes in plants. Our hypotheses were: 1) the responses of circRNAs are divergent in tomato seeds germinated under control and high temperatures; 2) circRNAs might play roles in tomato seeds in response to high temperature by regulating their parental genes. Therefore, we tried to identify the circRNAs in tomato seeds germinated under control and high temperatures, to determine differences in their expression, and possible regulation of the parental genes. This study can uncover the key circRNAs responding to heat stress during tomato seed germination. The outcome will have potential implications for understanding the function of this newemerging non-coding RNA in plants subjected to abiotic stresses.

sufficient for further analyses. Then, the seeds were immediately frozen in liquid nitrogen and stored at $-80^{\circ} \mathrm{C}$ before circRNA sequencing. There were three replications for each treatment.

The total RNAs of the six seed samples were extracted using an RNAprep Pure Plant kit (Tiangen Biotech, Beijing, China). The quality of total RNAs was detected by (Thermo Fisher Scientific, San Jose, USA) and Qubit 2.0 (Invitrogen, Carlsbad, USA) and Agilent 2100 (Agilent Technologies, Palo Alto, USA). Total RNAs with absorbances $\mathrm{A}_{260} / 280 \geq 1.8, \mathrm{~A}_{260} / 230 \geq 0.5$, concentration $\geq$ $65 \mathrm{ng} \mathrm{mm}^{-3}, \mathrm{RIN} \geq 7.0$, and $28 \mathrm{~S} / 18 \mathrm{~S} \geq 1.0$ were regarded as qualified. Then, $2 \mu \mathrm{g}$ RNA per sample was used to remove rRNA using the $N E B N e x t^{\mathbb{R}}$ rRNA depletion kit (Illumina, San Diego, CA, USA). The rRNA-depleted RNA was incubated at $37^{\circ} \mathrm{C}$ for $1 \mathrm{~h}$ using $20 \mathrm{U}$ of RNase $\mathrm{R}$ (Epicentre, Madison, WI, USA) to remove linear RNA. The six libraries were constructed using the $N E B N e x t^{\mathbb{B}}$ Ultra $^{\mathrm{TM}}$ small RNA sample library preparation kit for Illumina $^{\circledR} \quad(N E B)$. Fragmentation of circRNAs was performed by adding fragmentation buffer. The first strand cDNA was synthesized using a random hexamer primer with the short fragment as the template. The second strand cDNA was synthesized by adding dUTPs, DNA polymerase I, RNase $\mathrm{H}$, and buffer containing $10 \mathrm{mM}$ $\mathrm{Mg}^{2+}$ and $50 \mathrm{mM} \mathrm{Cl}^{-}$. Cohesive ends were converted into blunt ends using T4 DNA polymerase and Klenow DNA polymerase. NEBNext Adaptors with hairpin loop 
structures were ligated after the adenylation of 3 ' ends. The library fragments were purified using AMPure XP beads (Beckman Coulter, Beverly, USA). The second strand cDNA containing U was degraded using USER enzyme (NEB). Finally, PCR was performed to obtain the circRNA library.

Preliminary quantitative analysis was performed using Qubit 2.0. Then, the insert size of the library was detected using an Agilent 2100 bioanalyzer. Accurate quantitative analysis of the effective concentration of the library was conducted using quantitative (q)-PCR. The library was qualified and used for sequencing when the insert size was approximately $220 \mathrm{bp}$ and the effective concentration of the library was $>2 \mathrm{nM}$. The six qualified samples $(\mathrm{C} 1, \mathrm{C} 2$, and $\mathrm{C} 3$ as the control library and $\mathrm{H} 1, \mathrm{H} 2$, and $\mathrm{H} 3$ as the high-temperature library) were sequenced using the Illumina Hiseq X-Ten platform (Biomarker Technologies, Beijing, China).

Identification and expression of circRNAs: After sequencing, a saturation test of sequencing data was performed to ensure that the data were sufficient. The raw reads were processed by removing the junk reads with adaptors, ploy-Ns and low quality to obtain clean reads. The quality score could reflect the probability of base calling error $\left(\mathrm{Q}_{\text {-score }}=-10 * \log _{10} P\right)$. GC content and $\mathrm{Q}_{30}$ of the clean reads were checked. Clean reads with high quality were obtained and mapped to the reference genome sequence of Solanum lycopersicum (ftp://ftp. ensemblgenomes.org/pub/release32/plants/fasta/solanum lycopersicum/dna). CircRNAs were identified using Find circRNAs software that is able to identify circRNAs in a genome-wide range. Moreover, circRNAs were identified using the Plant circRNA detection tool (Chen et al. 2016). CircRNAs were taken into account when 1) GU/AG was on the sides of splice site; 2) a clear breakpoint could be detected; 3) two mismatches were present; 4) a breakpoint appeared in the position within 2 nucleotides (nt);5) at least two reads supported the junction; and 6) the BLAST score to the right position of the short sequence was $\geq 35$ higher than that of other positions.

The number of junction reads determined the expression of circRNAs. Before comparing the expressions, the circRNAs with low expressions were removed using DESeq software. In detail, the counts per million (cpm) of circRNAs were calculated, and the circRNAs with cpm $>1$ and in three replicated samples at the same time were retained. The expressions of the remaining circRNAs with high quality identified in the seeds germinated at 26 and $36^{\circ} \mathrm{C}$ were compared using the DESeq $R$ package (1.10.1). The $P$ values were adjusted using the Benjamini-Hochberg correction method to control the false discovery rate (FDR). The circRNA was considered to be up- or down-regulated when $\mid \log _{2}$ (fold change) $\mid>1$ and $P$ value $<0.05$. The expression levels of circRNAs identified in the $\mathrm{C} 1, \mathrm{C} 2$, and $\mathrm{C} 3$ libraries and $\mathrm{H} 1, \mathrm{H} 2$, and $\mathrm{H} 3$ libraries were averaged as the final expression levels of circRNAs for the control and high temperatures, respectively.

Functional analysis of circRNA parental genes: Gene Ontology (GO) enrichment analysis for the parental genes of the circRNAs only existing in the seeds at high temperature was performed. Then, GO enrichment analysis for the parental genes of the differently expressed circRNAs was performed.

The accession number of the sequencing data is PRJNA416990 (SRP124145) at https://www.ncbi.nlm. nih.gov/Traces/study/?acc=SRP124145.

\section{Results}

The germination rate of Sufen No. 14 at the control $\left(26^{\circ} \mathrm{C}\right)$ and moderately elevated temperatures $\left(32^{\circ} \mathrm{C}\right)$ showed no significant change and it was almost $100 \%$ after $60 \mathrm{~h}$ of incubation (Fig. 1). However, compared to the control, the germination rate at $36{ }^{\circ} \mathrm{C}$ significantly decreased. The germination rate of the seeds at $40{ }^{\circ} \mathrm{C}$ remained zero during the whole incubation period (Fig. 1).

The seeds germinated at $26^{\circ} \mathrm{C}$ and $36{ }^{\circ} \mathrm{C}$ were chosen for further experiments because the germination rate $9 \%$ at $36{ }^{\circ} \mathrm{C}$ was significantly different from $100 \%$ at $26{ }^{\circ} \mathrm{C}$ after $60 \mathrm{~h}$ of incubation (Fig. 1). Moreover, the samples were taken after $24 \mathrm{~h}$ of incubation when the significant change in germination rates started to be observed (Fig. 1).

High-throughput sequencing identified 50073233 and 51475845 clean reads in the tomato seeds germinated under the control ( $\mathrm{C}$ library, $26^{\circ} \mathrm{C}$ ) and high temperatures ( $\mathrm{H}$ library, $36^{\circ} \mathrm{C}$ ), respectively (Table 1$)$. The GC content and quality score $30(\mathrm{Q} 30)$ of the clean reads were 49.67 and $91.86 \%$ in the $\mathrm{C}$ library and 47.98 and $90.92 \%$ in the $\mathrm{H}$ library, indicating that clean reads with high quality were obtained. There were 48529611 and 50070777 mapped reads in the $\mathrm{C}$ and $\mathrm{H}$ libraries (Table 1). The mapped ratios of the $\mathrm{C}$ and $\mathrm{H}$ libraries were 96.93 and $97.20 \%$, respectively (Table 1 ), indicating that the data utilization rate was reasonable and that the data were qualified for further analysis.

In total, 4164 unique circRNAs from 2410 parental genes were identified by Find circRNAs in tomato seeds. CircRNAs generated from different chromosomes in tomato and 597 unique circRNAs from $\mathrm{Chrl}$ accounted for the most changes $(14.34 \%)$ (Fig. $2 A)$. Of the identified circRNAs, $3302(79.30 \%)$ were produced from exons of protein-coding genes; $640(15.37 \%)$ were from intergenic regions; $222(5.33 \%)$ were produced from introns with that determined by Find circRNAs (Fig. 3A). Moreover, 2743 and 2401 circRNAs were identified in the $\mathrm{C}$ and $\mathrm{H}$ 
libraries, respectively, and 980 circRNAs were shared in both libraries (Fig. 3B).

To obtain the critical circRNAs in response to high temperature, 3416 circRNAs with low expression were removed, and the expressions of the remaining 748 circRNAs in the $\mathrm{C}$ and $\mathrm{H}$ libraries were compared. Compared to the control, 31 and 42 circRNAs were up- and down-regulated (Fig. 2B). In total, 2001 circRNAs were identified by a Plant circRNA detection tool with 279 circRNAs shared at high temperature, respectively (Fig. 1 Suppl.). The mostup-regulated circRNAs were S1_ciR3009 (6:40387259| 40387508, 3.95-fold), while the most-down-regulated circRNAs were S1_ciR2964 (6:36065972|36070035, 3.20-fold) (Table 1 Suppl). Among the circRNAs with significant changes in expression, 28 circRNAs existed

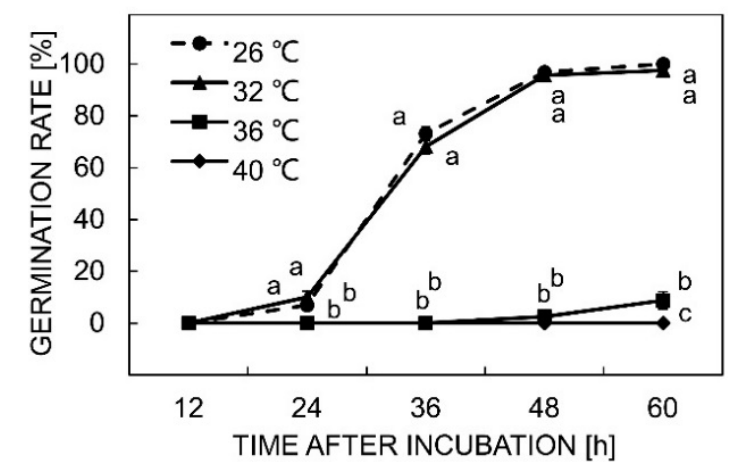

Fig. 1. The germination rate of tomato seeds incubated under control $\left(26^{\circ} \mathrm{C}\right)$ and high temperatures $\left(32,36\right.$, and $\left.40^{\circ} \mathrm{C}\right)$. Means \pm SEs, $n=8$, different letters indicate significant differences $(P<0.05)$.
Table 1. Data of circRNA sequences in the tomato seeds germinated under control (C library, $26{ }^{\circ} \mathrm{C}$ ) and high temperatures $\left(\mathrm{H}\right.$ library, $36{ }^{\circ} \mathrm{C}$ ). The results of the $\mathrm{C}$ library are the average of the $\mathrm{C} 1, \mathrm{C} 2$, and $\mathrm{C} 3$ libraries and the results of the $\mathrm{H}$ library are the average of the $\mathrm{H} 1, \mathrm{H} 2$, and $\mathrm{H} 3$ libraries. Mapped reads indicate that the reads could be mapped to the reference genome, unique mapped reads indicate that the reads could be mapped to the single position in the reference genome, and multiple mapped reads indicate that the reads could be mapped to more than one position in the reference genome.

\begin{tabular}{lll}
\hline Library & C & H \\
\hline Clean reads number & 50073233 & 51475845 \\
Mapped reads number & 48529611 & 50070777 \\
Mapped ratio & $96.93 \%$ & $97.20 \%$ \\
Unique mapped reads number & 38783010 & 42933978 \\
Unique mapped ratio & $77.51 \%$ & $83.23 \%$ \\
Multiple mapped reads number & 9746601 & 7136798 \\
Multiple mapped ratio & $19.42 \%$ & $13.97 \%$ \\
\hline
\end{tabular}

only in the C library, and 15 circRNAs existed only in the H library (Table 1 Suppl.).

According to the $\mathrm{GO}$ enrichment analysis, the parental genes of the circRNAs only existing in seeds at high temperature were primarily involved in the metabolic process, cellular process, catalytic activity, binding, and so on. Moreover, the parental genes of the circRNAs with different expressions between two libraries mainly played roles in the Golgi apparatus, intracellular protein transport, DNA methylation, chromatin silencing, Ran GTPase binding, and so on.
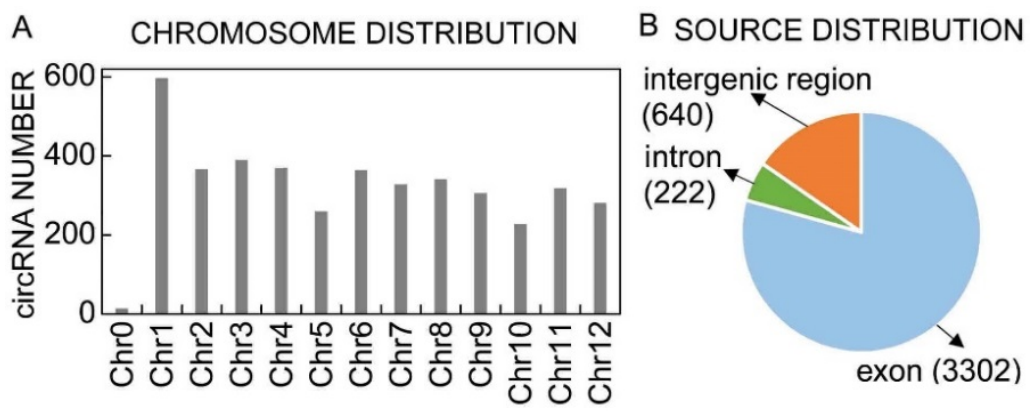

Fig. 2. Chromosome distribution $(A)$ and source distribution $(B)$ of the circRNAs identified in the tomato seeds. The circRNAs that were not distributed in $\mathrm{Chr} 1$ to $\mathrm{Chr} 12$ were grouped to $\mathrm{Chr}$.

\section{Discussion}

Abiotic stresses have multiple adverse effects on the growth and development of plants (Mittler 2006). Among the abiotic stresses, high temperature due to global warming has become an urgent issue for agricultural production, which has attracted increasing attention in recent years (Sung et al. 2003, Wahid et al. 2007).

High temperature may cause delayed seed germination and a significant reduction in final germination rate, leading to non-uniform seedlings, poor crop performance, and reduced yield (Jain et al. 2006, Wahid et al. 2007, Guan et al. 2009). In this study, the germination rate of tomato seeds was practically the same at 26 and $32^{\circ} \mathrm{C}$, but significantly lower at $36^{\circ} \mathrm{C}$. In accordance, Wahid et al. (2007) and Fatemeh et al. (2010) showed that the seed 
germination of tomato is detrimentally affected when the ambient temperature exceeds $35^{\circ} \mathrm{C}$. The visible signal that germination is complete is generally the breakthrough of the structures surrounding the embryo by a radicle (Bewley 1997). We found that the germination rates of the seeds at 26 and $36{ }^{\circ} \mathrm{C}$ were significantly different already after incubation for $24 \mathrm{~h}$, even though most of the seeds

\section{A}
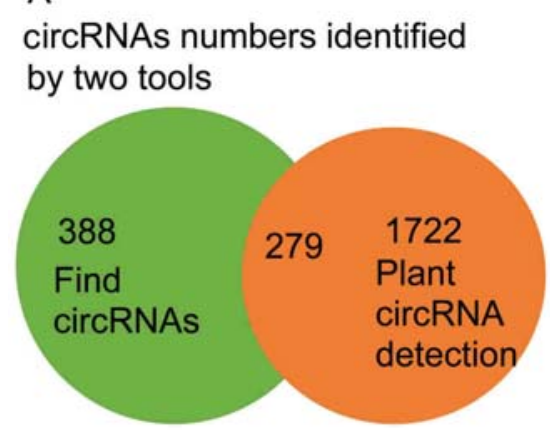

did not show visible radicles (Fig. 1). Many cellular and metabolic events are known to occur before the completion of germination (Bewley 1997). Therefore, a $24 \mathrm{~h}$ incubation period of tomato seeds was employed here when the difference between the temperature treatments was observed.

\section{B}

circRNAs numbers in $\mathrm{C}$ and $\mathrm{H}$

library

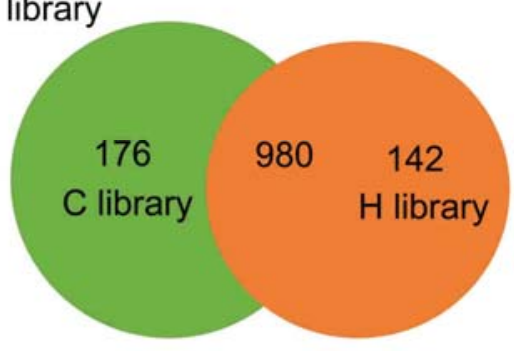

Fig. 3. CircRNA numbers identified by Find circRNAs and Plant circRNA detection tool $(A)$ and circRNA numbers in the tomato seeds germinated under control $\left(\mathrm{C}\right.$ library, $\left.26^{\circ} \mathrm{C}\right)$ and high temperatures $\left(\mathrm{H}\right.$ library, $\left.36^{\circ} \mathrm{C}\right)(\mathrm{B})$.

The large number of circRNAs with unique characteristics have made them a significant class of noncoding regulators that are worthy of more attention (Glazar et al. 2014). Few circRNAs were identified in plants compared to animals (Memczak et al. 2013, Guo et al. 2014, Ye et al. 2015, Meng et al. 2016). A comprehensive detection and genome-wide identification of circRNAs is an important step (Gao et al. 2015). Therefore, to obtain the circRNAs responding to high temperature, six small RNA libraries from tomato seeds germinated at 26 and $36{ }^{\circ} \mathrm{C}$ (three replications for each treatment) were constructed and sequenced. In total, 4164 circRNAs were identified, which uncovered the widespread occurrence of circRNAs in tomato and enriched the number of circRNAs detected not only in tomato but also in plants. In accordance with Zuo et al. (2016), different chromosomes produced different circRNA numbers, and exonic circRNAs accounted for the most, while intronic circRNAs accounted for the least (Fig. 2). This indicated that circRNAs in tomato were generated from different chromosomes and diverse genomic regions. Moreover, 1421 circRNAs existed only in the H library (Fig. 3B), indicating that these circRNAs were specific in tomato seeds incubated at high temperature.

On one hand, some circRNAs were previously identified in both tomato seeds and fruits, such as S1_ciR18 (1:958670|964383), S1 ciR88 (1:4383296|4383704) and S1_ciR2702 (5:22630066|22635161). On the other hand, some circRNAs found in this study were identified for the first time in tomato, such as S1 ciR1 (1:13177|13661), S1 ciR2 (1:35749|37729) and S1 ciR3 (1:383290|383547). This suggested that certain circRNAs showed tissuespecific expression patterns, which was consistent with the results of Lu et al. (2015) and Sablok et al. (2016).
Wang et al. (2017) reported that 62 circRNAs showed significant differences in wheat leaves between the polyethylene glycol treated and control plants, indicating specific roles of circRNAs in the dehydration stress regulation. In our study, 31 circRNAs were up-regulated and 42 circRNAs were down-regulated in tomato seeds germinated at $36{ }^{\circ} \mathrm{C}$ compared to the control. This indicated the potential role of these circRNAs in response to high temperature and it is in agreement with the stressspecific expression pattern of certain circRNAs (Ye et al. 2015, Sablok et al. 2016, Zuo et al. 2016). CircRNAs have miRNA response elements that can interact with miRNAs and play roles as miRNA sponges (Hansen et al. 2013). CircRNAs can block the elimination of target mRNAs and thereby regulate gene expression by bonding with miRNAs (Valdmanis and Kay 2013). This suggested that the circRNAs might play important roles in tomato at high temperature by regulating their target miRNAs. However, the function of most circRNAs as miRNA sponges is still unknown because the majority of the target miRNAs were not found in this study, which was in accordance with Gao et al. (2015) and Zhang et al. (2014b). Moreover, circRNAs have been shown to act as positive regulators of their parental genes (Salzman et al. 2012, Memczak et al. 2013, Zhang et al. 2014a). Lu et al. (2015) found that the overexpression of a circRNA construct could decrease the expression of its parental gene. These results indicated that circRNAs might have diverse functions in the regulation of their parental genes in tomato seeds. However, the function of circRNAs in tomato seeds at high temperatures requires further experimental validation.

In conclusion, 4164 circRNAs were identified in the tomato seeds under control and high temperatures, and 1421 circRNAs specifically existed in the seeds at high 
temperature. The 73 circRNAs with different expressions at high temperature in comparison with the control might play crucial roles in response to high temperature. The parental genes of circRNAs only existing in seeds at high temperature were primarily involved in the metabolic process, cellular process, catalytic activity, and binding according to GO enrichment analysis. These results indicated that circRNAs might have diverse biological

\section{References}

Bewley, J.D.: Seed germination and dormancy. - Plant Cell 9: 1055-1066, 1997.

Chen, L., Yu, Y., Zhang, X., Liu, C., Ye, C., Fan, L.: PcircRNA finder: a software for circRNA prediction in plants. - Bioinformatics 32: 3528-3529, 2016.

Darbani, B., Noeparvar, S., Borg, S.: Identification of circular RNAs from the parental genes involved in multiple aspects of cellular metabolism in barley. - Front. Plant Sci. 7: 776, 2016.

Fatemeh, M., Thomas, B., Garye, H.: Seed treatment with Trichoderma harzianum alleviates biotic, abiotic, and physiological stresses in germinating seeds and seedlings. Phytopathology 100: 1213-1221, 2010.

Fyfield, T.P., Gregory, P.J.: Effects of temperature and water potential on germination, radicle elongation and emergence of mungbean. - J. exp. Bot. 40: 667-674, 1989.

Gao, Y., Wang, J., Zhao, F.: CIRI: an efficient and unbiased algorithm for de novo circular RNA identification. - Genome Biol. 116: 1-16, 2015.

Glazar, P., Papavasileiou, P., Rajewsky, N.: CircBase: a database for circular RNAs. - RNA 20: 1666-1670, 2014.

Grime, J.P., Campbell, B.D.: Growth rate, habitat productivity, and plant strategy as predictors of stress response. - In: Mooney, H.A., Winner, W.E., Pell, E.J., Chu, E. (ed.): Response of Plants to Multiple Stresses. Pp. 143-159. Academic Press, London 1991.

Guan, B., Zhou, D., Zhang, H., Tian, Y., Japhet, W., Wang, P.: Germination responses of Medicago ruthenica seeds to salinity, alkalinity, and temperature. - J. arid. Environ. 73: 135-138, 2009.

Guo, J.U., Agarwal, V., Guo, H., Bartel, D.P.: Expanded identification and characterization of mammalian circular RNAs. - Genome Biol. 15: 1-14, 2014.

Hansen, T.B., Jensen, T.I., Clausen, B.H., Bramsen, J.B., Finsen, B., Damgaard, C.K., Kjems, J.: Natural RNA circles function as efficient microRNA sponges. - Nature 495: 384-388, 2013.

Huang, C., Shan, G.: What happens at or after transcription: Insights into circRNA biogenesis and function. Transcription 6: 61-64, 2015.

Jain, N., Kulkarni, M.G., Staden, J.V.: A butenolide, isolated from smoke, can overcome, the detrimental effects of extreme temperatures during tomato seed germination. - Plant Growth Regul. 49: 263-267, 2006.

Jeck, W.R., Sharpless, N.E.: Detecting and characterizing circular RNAs. - Nat. Biotechnol. 32: 453-61, 2014.

Jeck, W.R., Sorrentino, J.A., Wang, K., Slevin, M.K., Burd, C.E., Liu, J., Marzluff, W.F., Sharpless, N.E: Circular RNAs are abundant, conserved, and associated with ALU repeats. RNA 19: 141-157, 2012.

Li, Z., Huang, C., Bao, C., Chen, L., Lin, M., Wang, X., Zhong, activities by regulating their parental genes and target miRNAs in tomato seeds germinated at high temperature. Our results enrich the number of circRNAs in plants and provide the first genome-wide identification of circRNAs in response to high temperature during the germination of tomato seeds. Moreover, it is the basis for further study on the biological function characterization of circRNAs in tomatoes under heat stress.
G., Yu, B., Hu, W., Dai, L., Zhu, P., Chang, Z., Wu, Q., Zhao, Y., Jia, Y., Xu, P., Liu, H., Shan, G.: Exon-intron circular RNAs regulate transcription in the nucleus. - Nat. struct. mol. Biol. 22: 256-264, 2015.

Lu, T., Cui, L., Zhou, Y., Zhu, C., Fan, D., Gong, H., Zhao, Q., Zhou, C., Zhao, Y., Lu, D., Luo, J., Wang, Y., Tian, Q., Feng, Q., Huang, T., Han, B.: Transcriptome-wide investigation of circular RNAs in rice. - RNA 21: 2076-2087, 2015.

Memczak, S., Jens, M., Elefsinioti, A., Torti, F., Krueger, J., Rybak, A., Maier, L., Mackowiak, S.D., Gregersen, L.H., Munschauer, M., Loewer, A., Ziebold, U., Landthaler, M., Kocks, C., Noble, F., Rajewsky, N.: Circular RNAs are a large class of animal RNAs with regulatory potency. - Nature 495: 333-338, 2013.

Meng, X., Li, X., Zhang, P., Wang, J., Zhou, Y., Chen, M.: Circular RNA: an emerging key player in RNA world. - Brief Bioinform. bbw045, 2016.

Mittler, R.: Abiotic stress, the field environment and stress combination. - Trends Plant Sci. 11: 15-19, 2006.

Mueller, L.A., Solow, T.H., Taylor, N., Skwarecki, B., Buels, R., Binns, J., Lin, C., Wright, M.H., Ahrens, R., Wang, Y., Herbst, EV., Keyder, E.R., Menda, N., Zamir, D., Tanksley, S.D.: The SOL Genomics Network. A comparative resource for Solanaceae biology and beyond. - Plant Physiol. 138: 1310-1317, 2005.

Sablok, G., Zhao, H., Sun, X.: Plant circular RNAs (circRNAs): transcriptional regulation beyond miRNAs in plants. - Mol. Plant 9: 192-194, 2016.

Salzman, J., Gawad, C., Wang, P.L., Lacayo, N., Brown, P.O.: Circular RNAs are the predominant transcript isoform from hundreds of human genes in diverse cell types. - Plos ONE 7: e 30733, 2012.

Sung, D.Y., Kaplan, F., Lee, K.J., Guy, C.L.: Acquired tolerance to temperature extremes. - Trends Plant Sci. 8: 179-187, 2003.

Valdmanis, P.N., Kay, M.A.: The expanding repertoire of circular RNAs. - Mol. Theor. 21: 1112-1114, 2013.

Wahid, A., Gelani, S., Ashraf, M., Foolad, M.R.: Heat tolerance in plants: an overview. - Environ. exp. Bot. 61: 199-223, 2007.

Wang, P.L., Bao, Y., Yee, M.C., Barrett, S.P., Hogan, G.J., Olsen, M.N., Dinneny, J.R. Brown, P.O., Salzman, J.: Circular RNA is expressed across the eukaryotic tree of life. - Plos ONE 9: e90859, 2014.

Wang, Y., Yang, M., Wei, S., Qin, F., Zhao, H., Suo, B.: Identification of circular RNAs and their targets in leaves of Triticum aestivum L. under dehydration stress. - Front. Plant Sci. 7: 2024, 2017.

Ye, C.Y., Li, C., Chen, L., Zhu, Q.H., Fan, L.: Widespread noncoding circular RNAs in plants. - New Phytol. 208: 88- 
$95,2015$.

Zhang, X.O., Wang, H.B., Zhang, Y., Lu, X., Chen, L.L., Yang, L.: Complementary sequence-mediated exon circularization. - Cell 159: 134-147, 2014.

Zhang, Y., Yang, L., Chen, L.L.: Life without a tail: new formats of long noncoding RNAs. - Int. J. Biochem. Cell B. 54: 338349, 2014.

Zuo, J., Wang, Q., Zhu, B., Luo, Y., Gao, L.: Deciphering the roles of circRNAs on chilling injury in tomato. - Biochem. biophys. Res. Co. 479: 132-138, 2016. 